\title{
Interactive comment on "Global analysis for periodic variations of gravity wave squared amplitudes and momentum fluxes in the middle atmosphere" by Dan Chen et al.
}

\section{Anonymous Referee \#2}

Received and published: 3 April 2019

Formal review of the manuscript

Global analysis for periodic variations of gravity wave squared amplitudes and momentum fluxes in the middle atmosphere

by Dan Chen et al.

The paper by Dan Chen et al. presents the temporal variation of square-root zonal average gravity wave squared temperature amplitudes (GWSTA) and the GW momentum flux (GWMF) based on 13 years of satellite data of the SABER instrument. A spectral 
analysis is done focusing on the annual, semiannual, terannual oscillation up to quasibiennial periods. The origin of these oscillations is interpreted in terms of different GW sources and the propagation pathways using a data set based on the combination of ECMWF data with GROGRAT ray-tracing. The paper is well written, the methods are adequately described and the results are properly discussed, therefore a publication in Annales Geophysicae is highly recommended. But nevertheless, some minor concerns should be addressed as indicated below before publishing.

Specific comments:

1) The authors focus on the annual, semiannual, terannual oscillation up to quasibiennial periods as dominating oscillations. From Fig. 1a it can be seen that there are also oscillations with periods around 7 and 13 years visible. Please have a short discussion on that topic as these oscillations have even larger amplitudes than the QBO signal. Due to the length of the time series of 13 years, those peaks should be discussed in terms of the possible resolution within the FFT.

2) The difference of the variance based on the dominating periods between the northern and the southern hemisphere is related to SSW by the authors. It would be worth to discuss also the influence of planetary waves here as there are in general huge differences on both hemispheres due to the different land sea distribution.

Technical comments:

- Page 7, line 28: Spectral amplitudes in Fig. 1.b different compared to Fig. 1 a, for a reader it would be more intuitive to use the same axes as the color bar.

- Please improve the figure captions. Suggestion: Add labels GWSTA and GWMF for Fig. 2, 3, 5, 7, 11

- Page 35, Fig11: The colorbars in Figure 11 are very different. For a better comparison between each oscillation the color scale should be adjusted as equal as possible. 
Interactive comment on Ann. Geophys. Discuss., https://doi.org/10.5194/angeo-2019-31, 2019.
ANGEOD

Interactive

comment 